\title{
In reality: a medical anthropologist's reservations about the viability of leprosy control within Primary Health Care
}

\author{
I BIJLEVELD \\ Keizersgracht 426, 1016 GD Amsterdam, The Netherlands
}

Received for publication 9 September 1981

\section{Introduction}

Since the International Conference on Primary Health Care (PHC) held in Alma-Ata in September 1978, PHC has enjoyed great vogue. The idea, however, clearly did not just then drop from the clear sky. For a number of years in many places around the world a small-scale approach within the health sector had already been applied, often experimentally. Discussion of PHC grew in intensity, however, only after the WHO adopted it as official policy, initially in 1975 and yet more emphatically in 1978. The enthusiasm that invariably accompanies a new trend was contagious. Flushed with excitement, the supporters of PHC now seem vulnerable to over-confidence: 'How could we have been blind for so long'!

The cautionary remarks which follow are in no way an assault on the concept, the ideology, of PHC. My intention is to deflate runaway optimism. If we contemplate fitting leprosy control into PHC programmes, we need to ascertain whether the special character of the illness (chronicity, disabling complications, fear and stigma) lends itself to such an undertaking. A period of attempts to integrate leprosy control within general health services lies just behind us - indeed in many countries such attempts have only recently begun in earnest. Perhaps we need to consider more carefully the lessons these past years may have to teach us.

Before addressing the specific question of whether PHC can accommodate leprosy control, however, we need to face the general issue of whether PHC promises a viable solution to the pressing health needs of developing countries. To a large extent my experience of PHC has been indirect; that is, whilst I was engaged in research on leprosy control. During my most recent fieldwork 
in North Sulawesi, Indonesia, however, PHC became of increasing concern to me because of the Indonesian government's outspoken commitment to it. Examples below are drawn therefore for the most part from my Indonesian experiences (1979-80).

\section{Definition and roots of PHC}

Some confusion surrounds the definition of PHC. For many it means health work at the base, i.e. in such institutions as health centres, health posts, dispensaries. This view contrasts PHC to secondary and tertiary health care: medical assistance from specialists, in hospitals and at other care centres, $c f$. p. $350 .{ }^{1}$ If we were to adopt this definition, then leprosy control in many areas of the world would already form a part of PHC; leprosaria (tertiary health care) have largely yielded to outpatient, ambulant services at health centres. The WHO's notion of PHC, however, involves far more (see 'Declaration of Alma-Ata' 1978). Of crucial importance to the WHO is the active participation of the community in attending to their own health needs. This in turn implies identification of health priorities by the community itself. In short, communitybased health care. Seeing that the WHO definition has been winning wide acceptance, it seems advisable for us to agree to refer to a network of health centres and dispensaries where career health personnel are employed as an area's basic health services, reserving the term PHC for a community-oriented, community-based health care system. A salient characteristic of PHC in this specific sense is that its efforts are directed primarily at the prevention of illness. In addition to treatment of common illnesses and light injuries, PHC prominently includes nutrition, safe water, basic sanitation, mother and child care, immunization, health education, and prevention and control of endemic diseases.

In essence PHC is no novelty. Not only did some such programmes precede Alma-Ata in practice, but the guiding principles of self-help and selfdetermination derive from a movement of considerable long standing, the community development movement which reached its peak during the 50s and 60s. It is therefore rather odd that PHC has suddenly begun to receive such attention. Within their development package, community development programmes frequently tackled health problems. A rich literature exists recounting the successes and setbacks of such programmes, a valuable source for presentday advocates of PHC. ${ }^{2,3}$

Community development programmes aspired to development across a broad front; innovations and improvements in technology, agriculture, education, health care and other fields were to proceed integrally. In contrast the term PHC suggests a predominantly health-oriented enterprise. That, however, is certainly not true. Theorists behind PHC understand full well how intimately health is related to general well-being: ultimately an integrated approach to 
development holds the most promise. Since the early 1970s Indonesia, for example, has propagated multi-sectoral village development through the training of 'cadres'. These 'cadres', local people, have not only PHC responsibilities but are supposed to be active as well in adult education and agricultural extension activities. In reality, however, one field of training comes before another and different sectors tend to receive different degrees of emphasis. Where the earliest initiative for establishing cadres has come from the ministry of health, PHC is likely to overshadow other sectors.

\section{The attractions of PHC}

Keeping in mind the community development programmes which were in effect the antecedents of PHC and also their controversial results, we might well ask why PHC today has become so popular. Three explanations occur to me. First, it seems that PHC is winning converts because it is economical, an inexpensive means towards achieving the goal of health care for everyone. The WHO is explicit that promotion of health at the base must be attuned to costs that the community and country can afford. For most developing countries this means applied medical technology and the use of less highly-qualified, largely unsalaried health workers. No government can run up great losses in this fashion and therefore, where resources are limited, PHC is an alluring proposition.

Secondly, it seems logical that participation of the community in identifying their own health priorities guarantees that PHC will succeed. Motivation is most keen when innovations accord with the felt needs of the community. Such thinking is staunchly supported by western intellectuals either interested in or actively involved with development tasks in the Third World. The process of democratization as it has unfolded in our own societies, particularly during the previous decades, serves them as a model which they regard as congenial for development elsewhere.

Thirdly, where previous attempts to improve health care have turned out to be disappointing, policy makers and high level administrators have usually had to bear the blame. Whatever their motives for promoting secondary and tertiary health facilities, prestige and profit included, it appears that no significant improvement of public health can be credited to these initiatives. Should PHC fail in the end, however, decision makers at the top will be able to displace responsibility from their shoulders on to local communities, for self-determination is the name of the game.

\section{PHC can not work miracles}

To translate the attractive ideology of PHC into a functional system involves, as numerous examples suggest, severe headaches. Programmes launched years ago 
still struggle for survival. What are the main obstacles? Sitting behind our desks drafting a master blueprint, what did we overlook? To start with, it must be said that global planning invariably runs the risk of seeming deceptively easy. The variety of cultures in the world should alert us to the implausibility that a single remedy for health problems is likely to apply in all settings. We have to deal with densely- and sparsely-populated regions, with concentrated and scattered settlement patterns, with hunters-and-gatherers and peasant societies, with subsistence farming and cash cropping, societies with a high degree of cohesion and others characterized by continual conflict. Can we pretend to be so naive that we think a single approach to public health will be best suited everywhere? To make this point as cogently as possible, let us examine more closely that last-cited dichotomy: cohesion versus conflict. All too often, despite warnings to the wise, we are inclined to view non-Western societies through the distorting lenses of Rousseauean admiration: the scene appears idyllic, centripetal forces work to generate uniformity and harmony. This is, however, an illusion. Many non-Western societies are even more fragmented than our own. Cultural anthropologists have occupied themselves with this phenomenon. A much-discussed theory asserts that peasant societies are charged with internal antagonisms: wherever the size of the pie (the public good) is limited and each individual strives to lay claim to as large a piece of that pie as he can, structural opposition is the consequence. ${ }^{4}$ Given that most of the Third World consists of peasant societies, PHC in many places will have to face conditions which rhyme poorly with the prospect of basic community co-operation - and community co-operation is by definition intended to provide PHC with its underlying support. Where social splintering occurs and individuals jealously guard their own special interests, community participation may be easy to talk about, but next to impossible to achieve. The theory of peasant societies and their inherent discord may be disputable and far from universally valid, yet anyone who has lived or worked in a peasant society will know at first hand that envy, anger and competition simmer just under the surface: centrifugal forces so often prevent community action from getting off the ground to achieve change.

Of course societies are rare where the law of each-man-for-himself reigns absolutely. It is nevertheless common to observe antagonisms gravitate into alliances so that factions emerge or, in more hierarchical societies, classes form. 'Community' in either situation is largely a facade. The community is made up of different groups with different interests, values and expectations. Every new action, every proposal, is likely to contain seeds of conflict. If one talks of community participation, this may in fact turn out to be no more than the concerted effort of one group which hopes to strengthen its own position by monopolizing the innovation at hand. In the most favourable of situations this group will have a powerful grip on other groups and can oblige their compliance. Isn't this, however, practically to wring the neck of the principle 
of self-determination? All too often in reality the dominant group sees to it that its people occupy key positions in the innovational programme; in this way the group manipulates whatever advantages might derive to serve its own ends. Should other groups attempt to resist such monopoly formation, whatever services the innovation may have been designed to render are brought more or less to a standstill.

Another reservation worthy of consideration: how seriously are we to take the principle of self-determination when it comes to identifying and proposing solutions to community health problems? Are felt needs at all likely to conform to the perceptions of outside experts? The WHO badly wants to emphasize preventive measures. Many researchers, myself included, have found that what villagers desire instead is above all the elaboration of curative services. Despite health education and extensive discussion in cadre programmes in North Sulawesi, PHC appears to wake little response because prevention fails to interest people - people in good health. Most village health insurance schemes quickly run into financial difficulty; even a token contribution as a regular monthly premium comes to feel exorbitant when the time to pay comes around and no one in the family is sick. That people can get sick from using contaminated drinking water is common knowledge, but somehow inert and academic, not directly alarming. Government subsidies for village development are sooner spent for constructing imposing churches than for pipes, filters and water tanks. Similarly, despite propaganda to advance sanitation through the construction of safe WCs and despite the offer of the government to make expertise and materials available, many people prefer instead to erect expensive stone walls in front of their homes: this visible status symbol appeals to them more than safe WCs and intangible promises of improved health. Additional examples abound. The fact of the matter is that only curative health care elicits direct public response, and then only curative health care of a recognizably good quality.

To make PHC more attractive and to increase the respect with which villagers regard their village health workers these workers are generally equipped with a battery of first aid medicines: pain killers, tablets against fever, diarrhoea pills, etc. Even so, villagers seem to lose interest soon enough. Likely as not such preparations can also be bought cheaply at any one of the local shops. Furthermore in all corners of the globe it is already sad but true that when people feel unwell they equate health care with injections which they (all too soon) can receive at the nearest health centre. Nothing less than the needle any longer satisfies them. Conclusion: the felt needs of villagers probably differ far from what PHC planners would wish them to feel.

Such a gap can have damaging consequences for health care. In opposition to original intentions it has been possible to observe a rather rapid commercialization develop within PHC programmes. Certain village health workers, encouraged by community members who want to see improved curative care 
available close to home, expand their activities. They dispense drugs beyond their competence and give forbidden injections for payment. A black market, one dysfunctional for public health, flourishes. Thus self-determination in the community, however admirable in principle, requires guidance at times.

At this point, moreover, we should not forget that PHC programmes are unlikely to begin in a total health care vacuum. Even in outlying districts people will already have identified local figures to consult in the event of discomfort or illness, either a traditional curer or illegal dispenser. The extent to which such figures will compete with and sabotage PHC programmes is, I feel, well worth investigation, but unfortunately beyond the scope of what I am now writing, see e.g. pp. $311-313 .^{5}$

\section{Leprosy and PHC}

Having made a number of general comments about PHC, I would like to turn to the advisability of attempting to include leprosy services in such a communitybased programme. The health authorities of developing countries do not by and large accord leprosy control a high priority in their national activities. After all, other illnesses and problems are statistically far more important, and urgent, e.g. malaria, nutrition, birth control. What about priorities at the community level? From culture to culture the situation will of course vary. Here by way of illustration I wish to present certain relevant findings in Western Province, Kenya. Interviews, during which a questionnaire was used, established that for villagers in the Wanga Districts leprosy was one of the foremost perceived health problems. A somewhat surprising result, for within my research area there was a relatively low leprosy prevalence, far lower in fact than in neighbouring areas. For various reasons, however, people were so fascinated, so preoccupied by this disease that the attention they paid to it could be described objectively as out of proportion to the public health danger which leprosy presented. Isolated instances of visible crippling, the leprosy beggar problem in a nearby large town, fear of infection - especially from open wounds, the lack of any effective traditional remedy, smouldering doubts about the curability of leprosy, dread of isolation (the traditional lot of a leprosy patient was to be abandoned in a hut along a major river, later modified to lifelong containment in a camp, and more recently to submission to a variety of unspoken rules restricting an ambulant leprosy patient's behaviour in society) - all these considerations help to clarify why the community finds leprosy a pressing public health menace. Thus, there is no consensus between community felt needs and the priorities of health planners and experts. Is there perhaps agreement about how the leprosy problem should be tackled? Not at all. Although in Western Province integration of leprosy services within general health care began years ago, community members continue to reject such an arrangement and express 
a preference for seeing leprosy patients consigned to a special, distant leprosy hospital where they would not endanger others, nor affront them with their disfigurement. ${ }^{*}$ This attitude 'let's get rid of them', is even at times openly propagated by community leaders, pp. 27-41.6 Were we to take the principle of self-determination which is supposed to be at the heart of PHC literally, then the community has a decided wish to see leprosy treated not at the primary level, but the tertiary level. Out-patient treatment at health centres is available solely by the intervention of outside authorities. Indeed, before we attempt any democratization of leprosy control there is serious work to be done changing the mentality of communities involved.

Yet more than information is needed to change attitude and behaviour here. To give substance to health education, the demonstration effect of sound treatment is required. For the foreseeable future, there is in my opinion far more chance of achieving such sound treatment at health centres with career personnel who are more readily available for training and supervision than are dispersed village health workers. Even if we limit village health workers to routine leprosy chores and specify that all suspected new cases and all known patients with complications are to be referred to the health centre, I am left with doubts about the value of the contribution which they will be able to make. Can we realistically afford to entrust village health workers with administering multi-drug therapy - an 'unavoidable' step, it would appear, in the near future - when we have not yet demonstrated our ability to manage even simple DDS distribution satisfactorily through established health facilities? PHC depends upon the goodwill and devotion of the village health worker. In addition to these personal attributes which will in reality vary with the individual, health centre staff can be expected to possess some measure of career discipline and professional responsibility.

People will argue, I know, that leprosy control through village health workers has the advantage of the worker's closeness, literal and figurative, to the patients whom he treats and that within the village there is thus more possibility for exercising social control on the patient to continue with treatment as prescribed. Should inadequate patient compliance be the cause of disappointing leprosy control results such arguments might strike me as compelling. To date, however, successive studies have demonstrated that first and foremost poor staff compliance accounts for falling short of essential goals. ${ }^{7,8}$ At the risk of repeating myself: who can assure us that the job performance of an unpaid PHC village worker will be any improvement upon that of a salaried health centre nurse? Conscientious supportive supervision is crucial for effective leprosy control. Dependable supervision of widely dispersed village health

* Concrete behaviour displays much more tolerance and support for individual leprosy patients; what concerns us here for the sake of argument, however, is the stereotype of 'the leprosy patient' which affects the opinions people express. 
workers will invariably present more difficulties than supervision of health centre staff. Rather than plunge into experimentation with leprosy control as a component of PHC, I would urge intensifying efforts at improving integrated leprosy control within general health care delivery systems.

Often I have been told by health administrators that leprosy patients themselves would prefer receiving treatment as close to home as possible, from their village head, for example, or once PHC could be established, from a village health worker. A health centre is a facility which treats people from an extensive geographical region. Here, the story goes, leprosy patients feel less welcome. Experience, however, has taught me differently. Should a leprosy patient desire treatment close by, invariably he is mutilated or in some way conspicuous to the public eye. Patients with less obvious leprosy, the majority thus, do not necessarily put a high premium on services near at hand, especially not in societies where there is considerable stigma. The relative anonymity of the health centre, where a range of diseases is treated, even if this anonymity may be over-rated, is likely to be more acceptable to a new leprosy patient than the exposure which receiving medication in his own village must necessarily bring along with it.

\section{The Village K-: one of many examples}

The first PHC pilot projects in Indonesia took place in the early 1970s in Java. In time PHC became a leading objective within the Ministry of Health. Nurses' training, for example, was converted from a clinical to a public health approach to prepare student nurses for assuming posts in rural villages. (This revision in contrast to the education of doctors which remains largely clinical and poorly co-ordinated with community health development goals.) The example of cadre formation in Java was subsequently tried out in selected areas on other islands, North Sulawesi among them.

In North Sulawesi the initiative for a PHC project often comes from the sub-district health centre instead of from within the village itself as in Java. In the village $\mathrm{K}$ - it was the visiting health centre doctor, a man of extraordinary vitality, who proposed such a project, speaking about it for the first time from the pulpit of the largest church in the area, the Reformed Church. K- is part of the Minahasa Regency, a Christian enclave in Moslem Indonesia. In recent years many small splinter churches have been springing up. Belonging to a particular congregation has far-reaching consequences for all aspects of a person's life. Just as in pre- and post-World War II Holland, the bulk of a person's activities, however secular in nature these activities may be, take place together with fellow church members. It is easy to understand why the doctor chose to introduce his project within the church; it testifies to his appreciation of the processes at work in village life. Yet such a beginning left him the task of trying 
to mobilize the entire village despite church factionalism. He managed at any rate to insist on inclusion of 'cadres' from weaker church groups but it is relevant here to report that selection of 'cadres' finally took place not through election by fellow villagers but by appointment by the village head. An element of political factionalism was therefore also latent in the PHC programme in Kfrom its inception. Because the village was not unanimously in support of the village head, 'cadres' came predictably from the ranks of those he trusted.

For some months periodic day-long training sessions were held in $\mathrm{K}$ - at which 'cadres' were familiarized with the goals of public health and taught elementary techniques of health education and symptom-oriented first aid. Because $\mathrm{K}$ - is rather isolated, 'cadres' were provided with certain basic medicines.

The first case of leprosy in $\mathrm{K}$ - and the source of the disease which then rapidly spread was, according to popular legend, an immigrant Chinese shoemaker who arrived during the 1930s. At present leprosy appears to be claiming new victims at a reduced rate, but in the intervening years $\mathrm{K}$ - has become widely known for its many leprosy patients. With a population of little less than 2,000, there are 18 known leprosy patients now living in $\mathrm{K}$ - and tens of others who have taken up residence during the past in the provincial leprosy hospital.

Since 1972 there has been a specially trained leprosy worker attached to the nearest health centre. His responsibilities include treatment of patients from $\mathrm{K}$ - but his job performance has always left much to be desired. Some patients from $\mathrm{K}$ - prefer to travel a considerable distance to collect their supply of DDS at the leprosarium, either through official or unofficial channels: in this way they know their treatment will be unbroken. Others have found incidental sources of medicine for their leprosy, not all equally dependable. When the PHC programme in K- began, in 1977, leprosy was seen as one of the major village health problems. A decision was made to appoint a special village health worker for leprosy. We would do well, I feel, to question the motive behind this decision. Solution of other leading health problems was to be entrusted to 'cadres' who shared similar responsibilities and training. Only leprosy was singled out for a special health worker, and, what is more, this worker turned out to be himself a local leprosy patient! Many rationalizations for such an approach are plausible, such as a man who himself has the disease will sooner be trusted by other patients, will have more sympathy and thus more motivation to help others. Nevertheless there is something undeniably suggestive of social discomfort if not actually stigma about the arrangements in K-: a separate service for leprosy within what is essentially designed as an integrated programme, and, in addition, the choice of a leprosy patient to perform the job.

How did PHC in K- fare? After what all agree was an encouraging and enthusiastic beginning, the project soon enough stalled. The village insurance 
scheme to raise funds for medicines (and incentives for 'cadres') undercut the popularity of the programme. Villagers, moreover, wanted more powerful medicines than 'cadres' were initially allowed to hand out. Under popular pressure, 'cadres' more and more began to play doctor, making independent judgments about what treatment to offer, departing from what they had learned during training and relying on their own 'experience' instead. When the health centre doctor tried to correct the situation, limiting medicines which the 'cadres' came to him to request, the 'cadres' could and did journey to the provincial capital where even medicines which legally require a prescription are readily for sale over the counter of some pharmacies. Competition among 'cadres' for supremacy, the surfacing of rivalries within the structure of the village, introduced further tension into the PHC programme.

Should we perhaps also attribute programme difficulties to social and economic circumstances in K-? Indeed, because of its altitude and poor soil, $\mathrm{K}$ - is officially a 'minus area'. Cultivation of cloves has made the Minahasa a boom region, but $\mathrm{K}$ - has been left out. There are clove trees but they return a meagre harvest. Some social scientists theorize that where a community has already undergone a certain social-economic 'take off', a community development programme such as a PHC project will have more chance of succeeding, pp. 257-268. ${ }^{9}$ People will be more accommodating, give more credence, and be prepared to make more sacrifices for advances in the health sector. Yet wealth, especially if it is private, by no means guarantees that people will be more receptive to communal programmes. If, say, people in K- were to prosper, it is just as likely that those with new spending power would turn to private doctors for their family health needs and remain cool towards government projects. After all, in general private doctors provide better services. And, what is more, to visit the public sector, one may risk loss of prestige. Prestige in the Minahasa is a powerful force in determining general behaviour, and healthrelated behaviour is no exception.

The death blow to PHC in K- descended when the founding doctor was transferred to a new location. A project not born from within the community (and how many, in truth, are?) will stand or fall according to the sustained input of its initiator. In Indonesia doctors usually wind up being reassigned from one health centre to another after relatively short tours of duty - a pattern of transience which necessarily erodes PHC projects by disrupting continuity of supervision and support. There is now a new doctor at the health centre, but her interest in community-based programmes has to date been exclusively verbal.

As far as leprosy care in the PHC project in $\mathrm{K}$ - is concerned, this component was short lived. The appointed patient-health worker found a job on a road repair project outside the village and none of the other 'cadres' troubled to assume his tasks. 'Cadres' all knew who leprosy patients were, knew some were not on treatment, knew others went long distances for their medicine, and yet... The leprosy control situation is, as it was before PHC burst on the scene, unsatisfactory. 


\section{Conclusions}

The village K- illustrates various reasons why PHC may fail to take root. Other attempts to found programmes in North Sulawesi have brought additional factors to light. Yet something even more basic, I am afraid, is preventing PHC from catching on in the Indonesian setting. If we take a hard look at the dynamics of PHC, shouldn't we admit that it can succeed only where society as a whole has become involved in a process of fundamental change? In theory a multi-sectoral approach, health being but one part of integral development, may often be preached, but in practice this seldom is realized. Why has community-based health apparently achieved so much in China? ${ }^{10}$ Because the entire society was being turned upside down. PHC was part of a general revolutionary current which radically altered income distribution and power relations within the village. In this process of arriving at a new consciousness, PHC is an essential component. For those who wish to introduce PHC into a stable social situation, however, I predict little hope for achieving their goal. It will seem alien, an intrusion. Whatever attempts are made to mobilize such a static community, real headway on a meaningful scale is extremely unlikely. At most small projects of peripheral importance may make some progress.

With the reservations which I feel concerning the prospects for PHC in general, it is hardly to be expected that I would be sanguine about proposals to add leprosy care to such projects. It would be fine for treatment, reliable treatment, to be brought closer to leprosy patients. It would be ideal to involve family members of leprosy patients in the patient's treatment if this took place at village level. A great deal could be gained from thus providing families with support during crises arising from the illness of one of their members. Leprosy control as currently organized too seldom offers an opportunity for needed family counseling. Yet, above all, we must remain realistic. If PHC is eventually to prove advantageous for leprosy control, essential preconditions must first be met: good training, systematic supervision and effective referral possibilities for diagnosis and complications. Until the staff of health centres are able to demonstrate their ability to perform basic leprosy control adequately, it would, I feel, be a wild stab in the dark to decentralize control work still further. Only once the health centre has become a dependable institution and social and economic conditions appear favourable in general for attempting PHC should we contemplate involvement of village health workers on a programmatic basis. The advantages which the system could then offer are considerable. Even perspectives for social and vocational rehabilitation, important activities which within the health centre system of integrated leprosy control are, understandably enough, usually neglected altogether, would be vastly more promising. Indeed, it is the multi-sectorial aspect of PHC which may some day make it possible to assist the social-economic development of the disabled within their home villages, leprosy patients among others.

There is a great deal that is appealing about PHC as an idea, and even about prospects in the future for the incorporation of leprosy control within 
community-based health programmes. The reality of the field situation as I have known it suggests, however, that for the present it would be premature and counterproductive to divert efforts or resources to this new approach. When and where a PHC project has managed to take root in a community and has, for some years, demonstrated that it is functioning effectively, the time will be ripe for an attempt to introduce leprosy control into the project.

\section{References}

1 Muller F. The Myth of Primary Health Care: case studies from Peru', (Paper prepared for IUAES Congress Symposium 'Anthropology and Primary Health Care', Amsterdam April 1981, publication by Royal Tropical Institute pending). 1981. Mechanic David. Medical Sociology, second edition, New York, 1978.

${ }^{2}$ Paul Benjamin D. (ed.) Health, Culture and Community: Case Studies of Public Reactions to Health Programs, Russell Sage Foundation, New York, 1955.

${ }^{3}$ Foster GM. 'Primary Health Care: Its Conceptual Ties to Community Development', (Paper prepared for IUAES Congress Symposium 'Anthropology and Primary Health Care', Amsterdam April 1981 - publication by Royal Tropical Institute pending).

${ }^{4}$ Foster GM. 'Interpersonal Relations in Peasant Society', Human Organization, 1960$61 ; 19(4): 174$.

5 Foster GM. 'Medical Anthropology and International Health Planning', In: Logan MH, Hunt EE. Jr. eds., Health and the Human Condition. North Scituate, Mass. 1978.

${ }^{6}$ Bijleveld Iman. Leprosy and Other Diseases in the Three Wangas: Community Thought Patterns About Health Care and Their Consequences for Emergent Patients; Royal Tropical Institute, Amsterdam, 1976.

7 Bijleveld Iman. Leprosy Care: Patients' Expectations and Experiences, a case study in Western Province, Kenya; Royal Tropical Institute, Amsterdam, 1977.

8 Bijleveld Iman. 'Who Says Leprosy Patients are Ashamed or Lazy?', 3rd International Workshop on Leprosy Control in Asia, Taipei, Taiwan; Sasakawa Memorial Health Foundation, Tokyo, Japan, 1981.

9 Wertheim WF. Evolutie en Revolutie, De golfslag der emancipatie, Amsterdam, 1971.

10 Sidel, Victor W. and Ruth Sidel. Serve the People: Observations on Medicine in the People's Republic of China. Boston: Josiah Macy Jr. Foundation, 1973.

\section{Further reading}

Bijleveld I. 'Community Participation in Leprosy Control and Other Health Programmes: An Anthropologist's Viewpoint', 2nd Int Workshop on Leprosy Control in Asia, Kathmandu, Nepal; Sasakawa Memorial Health Foundation, Tokyo, Japan, 1979.

van der Geest S. 'The Secondary Importance of Primary Health Care: impressions from Cameroon', (Paper prepared for IUAES Congress Symposium 'Anthropology and Primary Health Care', Amsterdam April 1981, publication by Royal Tropical Institute pending). 1981.

Iskandar, Poerwanta and Adriaan S Rienks. 'Primary and Indigenous Health Care in Rural Java: a comparison of process and contents', (Paper prepared for IUAES Congress Symposium 'Anthropology and Primary Health Care', Amsterdam April 1981, publication by Royal Tropical Institute pending). 1981.

WHO. Official Records, No. 223. Executive Board Fifty-Fifth Session. Part 1, Resolutions, Annexes. Geneva, 20-31 Jan 1975, No. 226, Twenty-Eighth World Health Assembly. Part 1, Resolutions and Decisions, Annexes. Geneva 13-30 May 1975.

WHO. Primary Health Care. Report of the International Conference on Primary Health Care, Alma-Ata, USSR, 6-12 September 1978. Geneva, 1978. 